\title{
PDC Bit Hydraulic \& Mud Rheological simulation to model pressure drop across Bit
}

\author{
Ong Kai Sheng ${ }^{1}$, Tamiru Alemu Lemma ${ }^{1, *}$, and Shazaib Ahsan ${ }^{1}$ \\ ${ }^{1}$ Universiti Teknologi PETRONAS, 32610 Seri Iskandar, Perak Darul Ridzuan, Malaysia.
}

\begin{abstract}
During fluid flow from larger to smaller diameter pipes, a drop in pressure is experienced. High pressure drop across bit indicated high energy loss in the hydraulic system and also a setback to ROP performance. This is inefficient and pressure pumps would have to be of bigger sizing to make up for the losses. Present form of pressure drop models is in terms of mud density, flow rate, and total flow area. The objective of this paper is focused on the analysis of CFD simulation and to propose optimized parameters for improved ROP. Single phase flow study of Yield Power Law mud rheology was simulated at bottom hole of horizontal section. Parametric study on mud rheology was carried using DOE. Design points of DOE were sampled mostly using Latin Hypercube Sampling and a few by Central Composite Design. It is found that Kriging Response Surface method generated the best regression model where the predicted values are closest to the observed values and has the lowest Maximum Relative Residual $(0.000336 \%)$. Inlet velocity and Power Index have significant effect on pressure drop. Consistency Index showed moderate effect while Yield Stress showed small effect to pressure drop. This research has proven that pressure loss model should take into account of mud rheology. Further research can be done with PDC bit rotation and its effect on mud behaviour.
\end{abstract}

\section{Introduction}

In the oil and gas industry, drilling cost and time are of major concerns. Operators of this field have their focuses centred on minimizing the overall drilling cost while maintaining safe practices and environmental friendly operations. Rate of Penetration (ROP) is a measure of drilling speed. Based on the relationship between drilling cost and ROP, it had been shown that maximizing the ROP will result in minimizing the drilling cost [1]. Studies have been done on factors affecting ROP. These factors are categorized into bit design parameters and operational parameters. Bit design parameters that significantly affect ROP are Junk Slot Area (JSA) and Bit Size (BS). Operational parameters are Weight on Bit (WOB), Rotation of drill bit (RPM), Hydraulic Horsepower (HHP), Flow Rate, Nozzle Size, and Mud Weight (MW) [2]. High ROP would generate high rate of cuttings and vice versa. The removal of cuttings is undeniably necessary so that the bit can be in direct contact with bottom hole formation and drill deeper and faster. Cuttings are removed as mud circulates to the top and

\footnotetext{
* Corresponding author: tamiru.lemma@utp.edu.my
} 
carries the cuttings along. When cuttings accumulate at bottom hole, Equivalent Circulating Density (ECD) increases and ROP decreases. Besides that, bit hydraulics plays an important role during drilling. Good bit hydraulics help jet through the formation, keep the PDC cutters cool and clean, and prevents the JSA and nozzles from clogging up and balling. Moreover, mud rheology plays a huge role in drilling as well. Two main mud properties that have direct impact to removal of cuttings are viscosity and gel strength. Mud viscosity and gel strength primarily suspense cuttings and effectively sweeps the cuttings out of hole.

There are many issues during operations like bit hydraulics is crucial for the removal of cuttings and cutting the PDC cutters. With poor bit hydraulics, PDC bit may face problems like bit balling and plugged nozzle. These phenomena are the obstruction of JSA and nozzles which are caused by poor cuttings removal away from the PDC bit. Another issue is high pressure drop across bit nozzles is an indication of energy loss in the hydraulic system. If it is inefficient, then the pressure pumps have to be of bigger sizing to make up for the losses. How does mud rheology affect pressure drop across bit nozzles? From the issues discussed, the objectives of this investigation comprises of (i) to develop a CAD and CFD model for a typical PDC bit, (ii) to develop regression models for the pressure loss around the PDC bit, and (iii) to analyze the CFD simulation result and propose optimized parameters for improved ROP. The scopes of study based on the objectives can be simplified as (i) PDC bit size of 8.5 inches with length of CAD model 5 times the diameter of PDC bit, (ii) Single phase flow, (iii) Flow rate: 100 to $1000 \mathrm{gpm}$ and (iv) Mud rheological parameters (Yield Stress, Consistency Index, and Power Index).

\subsection{Bit Hydraulics}

Bit Hydraulics plays important role in cuttings removal away from PDC bit. Poor hydraulics may cause bad cuttings removal away from PDC bit and results in many problems as mentioned earlier. Optimization of bit hydraulics is through maximizing bit horsepower or nozzle-jet impact force [3]. This brings about effective cuttings removal as the cuttings are removed as fast as they are generated [4]. Flow rate of mud has significant positive effect on cuttings removal away from PDC bit [5-9]. Increasing the annular velocity by increasing the flow rate decreases the cuttings bed height significantly. At constant mud flow rate, smallersized nozzles increase cutting transport velocity as they provide higher jet velocity at bottom hole. Two nozzles showed higher cuttings-transport ratio as compared to three nozzles.

This is because the two nozzles generated asymmetrical flow which result in higher jet velocity and improved cutting transport [10]. For a similar total flow area (TFA) of nozzles, higher number of nozzles improves cuttings removal away from PDC bit as more nozzles provide a more-uniform fluid low distribution [11]. Bigger face volume of bit is at higher risk of bit balling when drilling at low ROP. And lower face volume achieved maximum ROP without balling. There is no correlation between face volume, JSA, and cuttings removal away from PDC bit efficiency [12].

\subsection{Pressure drop across bit}

Previous studies on pressure drop models are summarized in Table 1. Pressure drop across a bit happens when mud flows through the bit nozzles. The mud experiences this drop in pressure simply because it moves from large diameter drill pipes into the small diameter bit nozzles. This is highly important for the optimization of drilling hydraulic with the objective of maximizing hydraulic horse power or impact force without neglecting effectiveness of cutting removal. This sudden loss of pressure can be calculated from mud weight along with various parameters and can be derived from potential or kinetic energy. 
Table 1. Previous studies on Pressure Drop Models. Where $\Delta P_{b}$ is bit nozzle jets pressure loss $\left(\mathrm{lb} / \mathrm{in}^{2}\right) ; H H P$ is horse power at bit (hp); $M W$ is mud weight (lb/gal); $V_{n}$ is velocity of mud (ft/s); $Q$ is flow rate of mud (gpm); TFA is total flow area of bit nozzles $\left(\mathrm{in}^{2}\right) ; C_{d}$ is coefficient of bit nozzles (0.95 or 1.00 or 1.03 unitless); $R O P$ is rate of penetration $(\mathrm{ft} / \mathrm{hr}) ; R P M$ is drill bit rotation per minute (rpm).

\begin{tabular}{|l|c|l|}
\hline Source & Equations & \multicolumn{1}{c|}{ Remark } \\
\hline$[13]$ & $\Delta P_{b}=\frac{(M W)\left(Q^{2}\right)}{11884\left(C_{d}^{2}\right)\left(T F A^{2}\right)}$ & $\begin{array}{l}C_{d}=0.8 \text { (Conventional Bit) } \\
C_{d}=0.95 \text { (Jet Bit) }\end{array}$ \\
\hline$[14-16]$ & $\Delta P_{b}=\frac{(M W)\left(Q^{2}\right)}{12032\left(C_{d}^{2}\right)\left(T F A^{2}\right)}$ & $C_{d}=1.03$ \\
\hline$[17]$ & $\Delta P_{b}=\frac{(M W)\left(Q^{2}\right)}{8795\left[(T F A)\left(e^{-0.832}\right)\left(\frac{R O P}{R P M}\right)\right]^{2}}$ & - \\
\hline
\end{tabular}

\subsection{Mud Rheology}

Mud type has small to moderate positive effect on cuttings removal away from PDC bit [18]. Different mud types lead to different bed consolidation. Conventionally, there are two types of mud which is either oil based or water based mud. The two mud properties that have direct impact on cuttings removal away from PDC bit are viscosity and density. Viscosity plays function of the suspension of cuttings which is crucial for cuttings removal away from PDC bit. Rheology is defined as the science of deformation and flow of matter [19]. To date, all fluids are classified as either Newtonian or Non-Newtonian. Several rheological models have been developed based on research over time. The Herschel-Bulkley model is also called the Yield Power Law (YPL) model, since it takes both a yield point (YP) and a power law development into account. Effectively, it is a combination of the Bingham and power law fluid models. The Herschel-Bulkley model is often used to describe oil-well drilling fluids, since it considers both a yield point and power law development with increasing shear rate. The yield point factor is due to gelling.

$$
\tau=\tau_{y}+k \dot{\gamma}^{n}
$$

The rheological characteristics of drilling mud such as plastic viscosity (PV) and YP and the flow behavior indicators such as $k$ and $n$, of drilling mud play an important role in cleaning of drill cuttings. These fluid properties, especially the low shear rate rheological properties that prevail at annular section between the drill pipe and borehole wall have a major impact on the cuttings removal efficiency of drilling mud.

\section{Methodology}

\subsection{CAD Model and Mesh independency study simulations}

To simulate flow around a drill bit at bottom hole, it is required to prepare CAD drawings. A typical drill bit of length and diameter of $1.12 \mathrm{~m}$ and diameter of $220 \mathrm{~mm}$, respectively, is drawn in ANSYS Modeler. This is followed by a drawing of a borehole of the same length and diameter. Both CAD drawings are then aligned together with the same axial axis. After that, the drill bit is subtracted from the borehole. This leaves an annulus through which the mud will flow from drill pipe passing through bit nozzles into the annulus and then flow away from the bit. After the CAD model is prepared, it is necessary to lay mesh on the model. The smaller the mesh size, the more accurate the results but longer simulation time. This calls for mesh independence study which is the optimization of simulations on various mesh sizes ranging from 0.01 to 0.004 element size. For the study, water is selected with default values 
of properties for faster simulation time. Once the solver parameters are settled, hybrid initialization method is initialized and followed by the run of calculation. This optimization aims to reduce needless simulation time and produce consistent results. Validity of the results can be justified with small percentage error of less than five percent amongst all convergence criteria. In ANSYS Fluent, the solver used is pressure-based with absolute velocity formulation running in steady state of time. Since this research only revolves around single phase flow, the multiphase model is turned off. Viscous model of realizable $k$-epsilon is selected with standard wall functions and default values for constants. Density of all muds are fixed to $1198 \mathrm{~kg} / \mathrm{m}^{3}$. Viscosity of the muds are governed by YPL. Inlet velocity is varied according to flow rate over a fixed TFA as in Table 2.

\subsection{Parametric Study Simulations}

In the parametric study simulations, various muds rheology which are governed by yield power law are collected from past studies and compiled into upper and lower bounds as tabulated in Table 3. These upper and lower bounds were inputted into ANSYS' Design of Experiments program. Latin Hypercube Sampling design is chosen as it brings about no two points of equal value. Central Composite Design is used as backup when some of LHS' design points do not show expected results.

Table 2. Varying flow rate and inlet velocity over fixed TFA for preliminary simulations.

\begin{tabular}{|l|c|c|c|c|c|c|}
\hline Flow Rate, $\boldsymbol{Q}(\mathbf{g p m})$ & 300 & 400 & 500 & 600 & 700 & 800 \\
\hline Inlet Velocity, $\boldsymbol{V}_{\text {in }}(\mathbf{f t} / \mathbf{s})$ & 35.30 & 47.03 & 58.79 & 70.55 & 82.31 & 94.07 \\
\hline
\end{tabular}

Table 3. Upper and lower bound mud rheology variables govern by Yield Power Law for parametric study simulations.

\begin{tabular}{|c|c|c|c|c|}
\hline Mud Rheology & Unit & Min. & Max & Average \\
\hline Volume Flow Rate, $q$ & $\mathrm{gpm}$ & 100 & 1000 & 550 \\
\hline Inlet Velocity, $V_{\text {in }}$ & $\mathrm{m} / \mathrm{s}$ & 2.5 & 40 & 21.25 \\
\hline Yield Stress, $\tau_{0}$ & $\mathrm{~Pa}$ & 0.35 & 12 & 6.175 \\
\hline Consistency Index, $K$ & $\mathrm{~Pa} . \mathrm{sn}$ & 0.031 & 9 & 4.5155 \\
\hline Power Low Exponent, $n$ & - & 0.2 & 0.9 & 0.55 \\
\hline
\end{tabular}

\section{Results and Discussions}

\subsection{Preliminary Simulations}

Based on mesh independence study, mesh size of 0.06 provides consistent results within optimal time. Hence, further simulations are conducted using this particular mesh size. Flow rate of muds ranged from 300 to $800 \mathrm{gpm}$. Inlet velocities were calculated over the fixed TFA of CAD PDC bit model. Inlet pressures were recorded while values for outlet pressures were offset away from outlet boundary. This is because direct collection of data from the outlet boundary may give inaccurate data.

When mud flow rate increases, the pressure drop across bit increases. However, both simulations on water based mud (WBM) rheology show smaller pressure drop across bit nozzles as flow rate increases as shown in Fig. 1(a). The smaller the shear stress $(\tau)$ of WBM, the smaller the pressure drop. Rheological properties have significant positive effect on pressure drop; up to 50\% reduction in losses. The pressure drop points for the present study are shown in Fig. 2(a). 


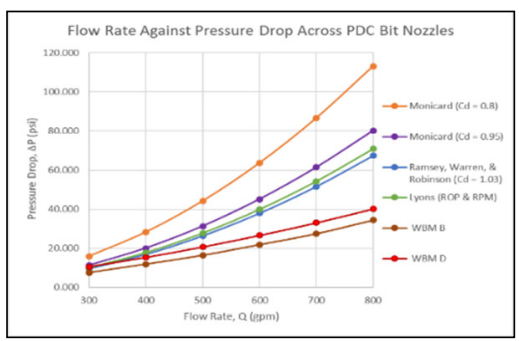

(a)

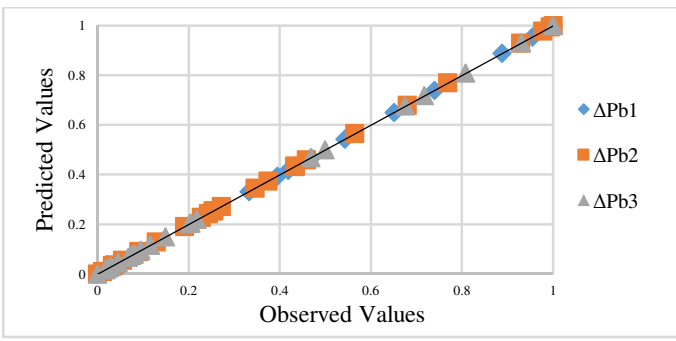

(b)

Fig. 1. (a) Graph of Flow Rate against Pressure Drop across PDC Bit Nozzles for WBM B \& D. (b) Goodness of Fit generated over Kriging Response Surface Types.

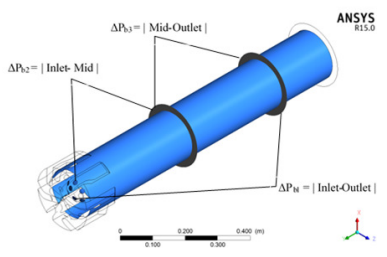

(a)
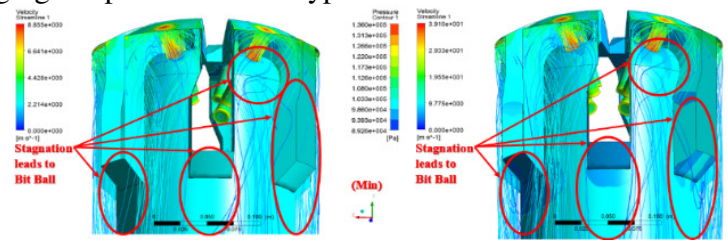

(b)

Fig. 2. (a) Pressure drop data points for this study. The outlet plane is offset away from the outlet to avoid boundary conditions and to obtain more accurate data. (b) Top and bottom represents overall input parameters at minimum and maximum each respectively.

\subsection{Parametric Study}

Type of DOE used to generate design points are mainly by Latin Hypercube Sampling with a few by Central Composite Design. This is because some of design points from LHS did not simulate expected result where the pressure drop is too small means invalid result.

To overcome this problem, CCD was used to sample additional design points and simulated results substituted those invalid results. Stagnation points are indications of bit balling as shown in Fig. 3(b). These points have very little or no velocity vector paths which means mud would be not circulated at these points. No proper mud circulation would definitely lead to bit ball despite high or low input parameters (emphasis on flow velocity). This can be concluded with bad geometry of the model and an optimized tweak to the geometry would eliminate the probability of bit balling.

\subsection{Regression Analysis}

There are a total of 4 types of Response Surfaces which are Standard Response Surface, Kriging, non-parametric Regression and Neural Network. The design points in all Goodness of Fit graphs are generally inline linearly. The blue and orange dots as shown in Fig. 1(b), which represents $\Delta \mathrm{P}_{\mathrm{b} 1}$ (Inlet-Outlet) \& $\Delta \mathrm{P}_{\mathrm{b} 2}$ (Inlet-Mid) respectively, are noticeably inline lineally and consistently over all 4 types of response surfaces.

However, the grey dots, which represents $\Delta \mathrm{P}_{\mathrm{b} 3}$ (Mid-Outlet), tend to be disoriented and dispersed in the Neutral Network. And, they appear to be closer to the linear line with Standard Response Surface Visually, Kriging and Non-Parametric Regression Response Surfaces would give the best Goodness of Fit. Between Kriging and Non-Parametric Regressions, Kriging yields the better results as shown in Table 4.

All response charts are plotted in terms of XYZ axes as depicted in Fig. 3 in which $V_{i n}$, $\Delta P_{b}$, and either $\tau_{o}, K$, or $n$ each, respectively. This is done as such to study the correlation 
between the parameters. Based on the generated charts, there are unnoticeable or insignificant differences between $\Delta \mathrm{P}_{\mathrm{b} 1} \& \Delta \mathrm{P}_{\mathrm{b} 2}$. This is because majority of pressure drop (Max $=265759$ $\mathrm{Pa})$ occurs nearer to the PDC bit while minority of pressure drop (Max $=13344 \mathrm{~Pa})$ occurs away from the bit. Minor pressure drop is approximately $5 \%$ of the total pressure drop across the bit.

$V_{i n} \& \Delta P_{b}$ have the strongest correlation in all cases. Around the bit, $\Delta P_{b 1} \& \Delta P_{b 2}$ increases as $V_{i n}$ increases. However, away from the bit, $\Delta P_{b 3}$ increases as $V_{i n}$ increases until $34 \mathrm{~m} / \mathrm{s}$ and then $\Delta P_{b 3}$ decreases. This shows that $V_{i n}$ has very strong positive effect on $\Delta P_{b}$ around the bit and slightly less effective away from the bit. To avoid large pressure drop across bit, flow velocity should be to a minimum $2.5 \mathrm{~m} / \mathrm{s}$.

$\tau_{o} \& \Delta P_{b}$ show the good correlation in all cases. $\Delta P_{b 1}, \Delta P_{b 2}, \& \Delta P_{b 3}$ increase as $\tau_{o}$ increases. $\Delta P_{b 1}, \Delta P_{b 2}, \& \Delta P_{b 3}$ peak when $\tau_{o}$ is $5.6 \mathrm{~Pa}$ and then $\Delta P_{b 1}, \Delta P_{b 2}, \& \Delta P_{b 3}$ decrease as $\tau_{o}$ increases. $\tau_{o}$ has positive and negative effect on $\Delta P_{b 1}, \Delta P_{b 2}, \& \Delta P_{b 3}$. The value of $\tau_{o}$ should be away from 5.6 Pa; either very small or large value. $\tau_{o}$ is better off at $11.25 \mathrm{~Pa}$; the higher the better.

$K \& \Delta P_{b}$ also show the good correlation in all cases. $\Delta P_{b 1}, \Delta P_{b 2}, \& \Delta P_{b 3}$ decrease slightly as $K$ increases until 2.5 Pa.s $\mathrm{s}^{\mathrm{n}}$ and then $\Delta P_{b 1}, \Delta P_{b 2}, \& \Delta P_{b 3}$ increase as $\tau_{o}$ increases. To minimize pressure drop across bit, $K$ value should be kept low; approximately 2.5 Pa. $\mathrm{s}^{\mathrm{n}}$.

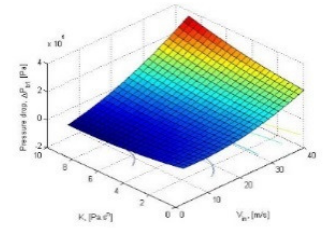

(a)

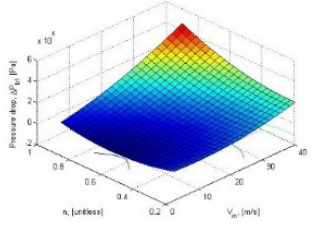

(b)

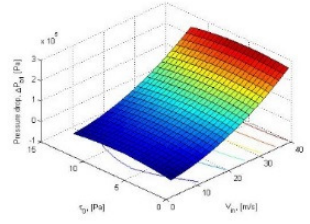

(c)

Fig. 3. (a) 3D Response Charts of $\Delta \mathrm{P}_{\mathrm{b}}$ against $\mathrm{K}$. (b) $3 \mathrm{D}$ Response Charts of $\Delta \mathrm{P}_{\mathrm{b}}$ against $\mathrm{K}$. (c) 3D Response Charts of $\Delta \mathrm{P}_{\mathrm{b}}$ against $\mathrm{K}$.

Table 4. Goodness of Fit details on various types of Response Surfaces.

\begin{tabular}{|c|c|c|c|}
\hline Kriging & $\boldsymbol{\Delta \boldsymbol { P } _ { \boldsymbol { b } 1 }}$ & $\boldsymbol{\Delta P}_{\boldsymbol{b} \mathbf{2}}$ & $\boldsymbol{\Delta \boldsymbol { P } _ { \boldsymbol { b } \mathbf { 3 } }}$ \\
\hline Coefficient of Determination (Best Value = 1) & 1 & 1 & 1 \\
\hline Adjusted Coefficient of Determination (Best Value =1) & 0 & 0 & 0 \\
\hline Maximum Relative Residual (Best Value = 0\%) & 0.000336 & 0.000302 & $5.73 \mathrm{E}-05$ \\
\hline
\end{tabular}

\section{Conclusion and Recommendations}

This research is about pressure drop across bit with respect to mud rheological parameters. Objectives were achieved with successful development of regression model of pressure drop across bit and optimization of parameters for improved ROP. Some important conclusions that can be drawn are: (i) Mud flow rate has significant effect to pressure drop across the bit. The lower the inlet velocity, the lower the pressure drop, (ii) Mud rheology parameters have been proven to show moderate effects to pressure drop across the bit. Power Index showed significant effect to pressure drop. Consistency Index showed moderate effect while Yield Stress showed small effect to pressure drop. This research has 4 input parameters and optimization analysis were done individually where the other 3 input parameters are kept at average values. Optimized parameters for reduction in pressure drop across bit and improvement in ROP: (Inlet Velocity $V_{\text {in }}=2.5 \mathrm{~m} / \mathrm{s}$, Yield Stress $\tau_{0}=11.25 \mathrm{~Pa}$, Consistency Index $K=2.5 \mathrm{~Pa}$.sn, Power Index $n=0.425$ ) and (iv) Simulation on mud rheology showed a reduction of about $50 \%$ in pressure drop across the bit as compared to existing models. Existing pressure drop models should be revised to include mud rheology parameters. 
The recommendations from this research is further research on the effect of drill bit rotation (RPM) and its effect on the mud behavior should be considered as it was not considered in previous study.

The Authors are thankful to Universiti Teknologi PETRONAS for facilities provided for the research.

\section{References}

1. A.T. Bourgoyne, Applied drilling engineering. (SPE (U.S.), Richardson, TX, 1991).

2. D. Hankins, S. Salehi, and F.K. Saleh, J. PETROL ENG 2015, 1 (2015).

3. H.A. Kendall and W.C. Goins, Jr., AIME SPE 219, 238 (1960).

4. B. Guo and G. Liu, Applied Drilling Circulation Systems: Hydraulics, Calculations and Models. (GPP., Burlington, MA., 2011).

5. S. M. Hussaini and J.J. Azar, SPE J. 23(01), 11 (1983)

6. P.H. Tomren, A.W. Iyoho, and J.J. Azar, SPE DRILL ENG 1(01), 43 (1986).

7. J. Li and S. Walker, SPE J. 6(04), 356 (1991).

8. H. Cho, S.N. Shah, and S.O. Osisanya, SPE ANTEC (2001).

9. K. Ravi and T. Hemphill, IADC SPE (2006).

10. K.M. Lim, and G.A. Chukwu, SPE WRM (1996).

11. G. Mensa-Wilmot and M. Fear, IADC SPE (2002).

12. M. Wells, T. Marvel, and C. Beuershausen, IADC SPE (2008).

13. R. Monicard, Drilling Mud and Cement Slurry Rheology Manual. (Springer, Netherlands, 1982).

14. L. H. Robinson, M. S Ramsey, AADE 01-NC-HO-31 (2001).

15. T. M. Warren, SPE DRILL ENG 4(04), 335 (1989)..

16. L. Robinson, AADE 10-DF-HO-26, 1 (2010).

17. W.C. Lyons, G.J. Plisga, M.D. Lorenz, Standard Handbook of Petroleum and Natural Gas Engineering (Third Edition) (GPP., Boston, 2016).

18. T. Nazari, G. Hareland, and J.J. Azar, SPE WRM (2010).

19. R. Darby, Viscoelastic Fluids. An Introduction to Their Properties and Behavior. (Marcel Dekker, New York, 1976). 